\title{
Auswirkungen des Klimawandels auf das Grundwasser und die Wasserversorgung in Süddeutschland
}

\author{
Ronja Fliß ${ }^{1}$ D $\cdot$ Christof Baumeister ${ }^{2}$ Thomas Gudera ${ }^{3} \cdot$ Mario Hergesell $^{4}$ Benjamin Kopp ${ }^{1} \cdot$ Jörg Neumann ${ }^{1}$. \\ Miriam Posselt ${ }^{1}$
}

Eingegangen: 19. Mai 2020 / Überarbeitet: 16. Oktober 2020 / Angenommen: 21. Dezember 2020 / Online publiziert: 22. Februar 2021

(c) Der/die Autor(en) 2021

\section{Zusammenfassung}

Für die öffentliche Trinkwasserversorgung in Süddeutschland wird größtenteils Grund- und Quellwasser genutzt. Der Kenntnis über die Entwicklung des Bodenwasserhaushalts und insbesondere der Grundwasserneubildung kommt somit eine große Bedeutung zu. Im Rahmen der Kooperation KLIWA (Klimaveränderung und Konsequenzen für die Wasserwirtschaft, www.kliwa.de) wurde die Entwicklung des Bodenwasserhaushaltes und der Grundwasserneubildung aus Niederschlag für den Zeitraum von 1951 bis 2019 für die Länder Baden-Württemberg, Bayern, Hessen und RheinlandPfalz mit dem Bodenwasserhaushaltmodell GWN-BW modelliert. Seit Ende der 1980er-Jahre zeigt sich ein Anstieg der mittleren jährlichen Temperatur in Süddeutschland. Die jährlichen Niederschlagssummen haben sich in der jüngeren Vergangenheit (seit 2003) reduziert und somit eine deutliche Verringerung der Grundwasserneubildung aus Niederschlag bewirkt. Gleichzeitig ist eine Zunahme des Trockenheitsindex (Anzahl an Tagen mit Füllung des Bodenwasserspeichers $<30 \%$ der nutzbaren Feldkapazität) zu erkennen. Häufiger werdende und länger anhaltende Trockenperioden stellen auch die Wasserwirtschaft zunehmend vor Herausforderungen und bedürfen der frühzeitigen Entwicklung von Handlungsoptionen, z.B. auf der Basis des hier vorgestellten „Trockenwetterdargebots“, um Nutzungskonflikte zu entschärfen.

Schlüsselwörter Wasserversorgung · Bodenwasserhaushalt · Grundwasserneubildung · Klimawandel · Handlungsoption · Anpassung

Zusatzmaterial online Zusätzliche Informationen sind in der Online-Version dieses Artikels (https://doi.org/10.1007/s00767021-00477-z) enthalten.

\footnotetext{
Ronja Fliß

ronja.fliss@1fu.bayern.de

Christof Baumeister

christof.baumeister@1fu.rlp.de

$\triangle$ Thomas Gudera

thomas.gudera@lubw.bwl.de

Mario Hergesell

mario.hergesell@hlnug.hessen.de

Benjamin Kopp

benjamin.kopp@lfu.bayern.de

Jörg Neumann

joerg.neumann@lfu.bayern.de

Miriam Posselt

miriam.posselt@lfu.bayern.de
}

1 Bayerisches Landesamt für Umwelt, Hans-Högn-Str. 12, 95030 Hof, Deutschland

2 Landesamt für Umwelt Rheinland-Pfalz, Kaiser-Friedrich-Str. 7, 55116 Mainz, Deutschland

3 LUBW Landesanstalt für Umwelt Baden-Württemberg, Griesbachstr. 1, 76185 Karlsruhe, Deutschland

4 Hessisches Landesamt für Naturschutz, Umwelt und Geologie, Rheingaustr. 186, 65203 Wiesbaden, Deutschland 


\title{
Impacts of climate change on groundwater recharge and water supply in southern Germany
}

\begin{abstract}
Public water supply in southern Germany relies to a high degree on the usage of groundwater and spring water. Thus, knowledge about the development of the soil water balance and especially groundwater recharge is important. Within the cooperation project KLIWA (Climate Change and Consequences for Water Management), the development of the soil water balance and groundwater recharge from precipitation are simulated from 1951 until 2019, applying the soil water balance model GWN-BW. An increase in the annual mean temperature in southern Germany since the late 1980s is apparent. The amount of precipitation has decreased in the recent past, thus reducing groundwater recharge by precipitation. Simultaneously, an increase of the drought index (number of days with soil water content $<30 \%$ of the available water capacity) is visible. An increase in frequency and duration of drought periods challenges water management and requires early action recommendations, e.g. based on the here presented "dry weather supply", to diminish resource conflicts.
\end{abstract}

Keywords Water supply $\cdot$ Soil water balance $\cdot$ Groundwater recharge $\cdot$ Climate change $\cdot$ Action recommendation $\cdot$ Adaption

\section{Einleitung}

In Süddeutschland stützt sich die öffentliche Trinkwasserversorgung zu einem vergleichsweise geringen Anteil auf die Nutzung von Oberflächenwasser und zum überwiegenden Teil auf die Nutzung von Grund- und Quellwasser (Baden-Württemberg 71\%, Bayern 90\%, Hessen $100 \%$, Rheinland-Pfalz 85\%) (Statistisches Bundesamt 2018). Die Grundwasserneubildung aus Niederschlag stellt dabei ein wichtiges Maß für die natürliche Regeneration der Grundwasserressourcen dar. Die quantitativen Auswirkungen des Klimawandels auf die Wasserversorgung sind daher in erster Linie abhängig vom jeweiligen regionalen Grundwasserdargebot, der regionalen Bedarfsentwicklung seitens der Bevölkerung und damit einhergehend auch der demographischen Entwicklung. Eine besondere Herausforderung für die Wasserwirtschaft, heute und in Zukunft, stellt die Sicherstellung des Spitzenwasserbedarfs der öffentlichen Trinkwasserversorgung in Trockenzeiten, bei gleichzeitig erhöhtem Bewässerungsbedarf seitens der Landwirtschaft, dar (LAWA 2017). Je nach Fragestellung und um Konflikten zwischen Trinkwassergewinnung und konkurrierenden Nutzungen vorzubeugen, gewinnen geeignete lokale bis überregionale Handlungsempfehlungen und Niedrigwassermanagementkonzepte erheblich an Bedeutung (LfU 2017). Diese müssen über ein rein reaktives Krisenmanagement (operative Maßnahmen) hinausgehen und bereits vorsorglich zur Abmilderung der Auswirkungen von Trockenzeiten gültige Planungen, Strategien und Maßnahmen einschließen. Um die nötige Akzeptanz und Wirksamkeit der Maßnahmen zu erreichen, sind diese jeweils abgestimmt auf die lokalen/regionalen Verhältnisse unter Einbeziehung aller vorhandenen Nutzer (u. a. Trinkwassergewinnung, Industrie/Gewerbe, Landwirtschaft, Naturschutz) gemeinsam zu entwickeln (LfU 2017).
Die Notwendigkeit wasserwirtschaftlicher Anpassungsmaßnahmen in Trockenzeiten wird durch die voranschreitende Erwärmung der Erdoberfläche seit dem Jahr 1850 sukzessive verstärkt (IPCC 2016; LfU 2017). So ist in den Messdaten der Lufttemperatur in den Bundesländern Baden-Württemberg, Bayern, Hessen und Rheinland-Pfalz seit dem Ende der 1980er-Jahre ein deutlicher Anstieg zu erkennen (Abb. 1a). Das Trockenjahr 2018, zugleich das wärmste Jahr in Deutschland seit Beginn der flächendeckenden Messungen im Jahr 1881, gefolgt vom zweitwärmsten Jahr 2019 (DWD 2020), hat sich erheblich auf den Grund- und Bodenwasserhaushalt ausgewirkt. Steigende Temperaturen führen bei ausreichendem Niederschlagsdargebot zu einer Zunahme der Verdunstung. Gleichzeitig haben sich die Niederschläge, vor allem während des hydrologischen Winterhalbjahres (November bis April), in den letzten Jahren (seit 2003) verringert (siehe Abb. 1 im Anhang, verfügbar als Zusatzmaterial der Online-Ausgabe dieses Beitrags). Als Folge ist die Grundwasserneubildung flächendeckend zurückgegangen (Kopp et al. 2018), was sich auch in der Entwicklung der Grundwasserstände und Quellschüttungen zeigt. So wurden bis zuletzt an vielen Messstellen in Süddeutschland vermehrt abnehmende Grundwasserstände und Quellschüttungen sowie auch neue Niedrigstwerte registriert (LfU 2019; HLNUG 2019; LfU RP 2019; LUBW 2019; KLIWA 2019a).

Im Rahmen des Kooperationsvorhabens KLIWA arbeiten die Bundesländer Baden-Württemberg, Bayern und Rheinland-Pfalz sowie der Deutsche Wetterdienst (DWD) länderund fachübergreifend zusammen, um die möglichen Auswirkungen und Konsequenzen der Klimaveränderung auf den Wasserhaushalt und die Grundwasserressourcen im Süden Deutschlands aufzuzeigen und Handlungsempfehlungen zu erarbeiten. Neben Untersuchungen auf Grundlage von WETTREG-Klimaprojektionen (KLIWA 2012, 2017) wurde, unter Beteiligung von Hessen, auf Grundlage des 
Bodenwasserhaushaltsmodells GWN-BW eine flächendeckende Langzeitsimulation des Bodenwasserhaushalts und der Grundwasserneubildung für die vier Länder von 1951 bis 2015 durchgeführt (KLIWA 2017; Kopp et al. 2018). Die kooperativ und methodisch einheitlich durchgeführten flächendeckenden Modellierungen mit GWN-BW ermöglichen Aussagen zur länderübergreifenden Entwicklung der Grundwasserneubildung aus Niederschlag im süddeutschen Raum.

Mit der nun erfolgten Aktualisierung der Langzeitsimulation bis einschließlich 2019 liegen flächendeckend Daten der Wasserhaushaltskomponenten für die Bundesländer Baden-Württemberg, Bayern, Hessen und RheinlandPfalz einschließlich der letzten beiden Trockenjahre 2018 und 2019 vor. Die Auswertung der meteorologischen Eingangsdaten Luftemperatur und Niederschlag, der Wasserbilanzgrößen Verdunstung, Gesamt- und Sickerwasserabfluss und der Grundwasserneubildung aus Niederschlag ermöglicht Trendaussagen zur regionalen Langzeitentwicklung und erlaubt eine statistische Einordnung der letzten Jahre im Kontext des globalen Klimawandels. Trockenjahre liefern zudem wichtige Erfahrungswerte für den zukünftigen Umgang mit den zu erwartenden Auswirkungen des Klimawandels (Klimaanpassung) und sind damit insbesondere für eine nachhaltige Bewirtschaftung und Sicherstellung der Wasserversorgung von grundlegender Bedeutung. Zusätzlich können anhand der Auswertungen zum Trockenheitsindex Aussagen über die räumliche und zeitliche Ausdehnung von erhöhtem Trockenstress für die Vegetation getroffen werden (WaBoA 2012).

\section{Methodik}

Das angewendete Bodenwasserhaushaltsmodell GWN-BW ist ein modular aufgebautes, deterministisches und flächendifferenziertes Modell zur Berechnung der tatsächlichen Verdunstung, zur Simulation des Bodenwasserhaushaltes und der unterhalb der durchwurzelten Bodenzone gebildeten Sickerwassermenge (Gudera und Morhard 2015). In den einzelnen Teilmodulen werden physikalisch-basierte und konzeptionelle Ansätze verwendet.

Im Interzeptionsmodul wird die Interzeption von Niederschlagswasser auf versiegelten Flächen und in der Vegetationsbedeckung berücksichtigt, wobei je nach Art der Vegetation diese in bis zu sieben Schichten aufgeteilt wird (Armbruster 2002). Das Transpirationsmodul basiert auf Penman-Monteith und bildet die Einflüsse von Bedeckungsgrad (Pflanzen- bzw. Bodenverdunstung) und Bodenfeuchte in separaten Schritten ab (Modellansatz „Persephone“). Die Parametrisierung von landnutzungsabhängigen Parametern wie minimalem Bestandswiderstand (Faktor zur Berechnung der Evapotranspiration) oder Blattflächenindex
(Dichte eines Pflanzenbestands) folgt dabei den phänologischen Entwicklungsstadien der Vegetation, deren Eintrittszeitpunkte wiederum auch witterungsabhängig über Temperatursummen berechnet werden (Morhard 2009). Zusätzlich wird die aktuelle Transpiration reduziert, wenn pflanzenverfügbares Wasser nicht ausreichend im Bodenspeicher vorhanden ist.

Mit der nutzbaren Feldkapazität des effektiven Wurzelraums (nFKWe) wird der maximale, pflanzenverfügbare Wassergehalt des Bodenspeichers beschrieben. Das Niederschlag-Abfluss-Modell HBV (Bergström 1976) diente dem Bodenmodul zur Simulation der Auffüllung und Entleerung des Bodenspeichers als Grundlage. Für grundwasserbeeinflusste Flächen wird der kapillare Aufstieg entsprechend den Angaben der bodenkundlichen Kartieranleitung (Adhoc-Arbeitsgruppe Boden 2005) berechnet.

In Gebieten mit vernachlässigbar schnellen lateralen Abflusskomponenten (nicht-reliefierte, gut durchlässige Porengrundwasserleiter) entspricht die berechnete Sickerwasserrate näherungsweise der Grundwasserneubildung aus Niederschlag. In Gebieten mit schnellen lateralen Abflusskomponenten (reliefierte Festgesteinsgebiete und gering durchlässige Porengrundwasserleiter) wird für die Berechnung der Grundwasserneubildung eine Reduktion der berechneten Sickerwasserrate mittels Baseflow-Index (BFI) erforderlich. Durch den BFI erfolgt eine Separierung in die Abflusskomponenten Direktabfluss und Grundwasserneubildung auf unversiegelten Flächen (Gudera und Morhard 2015). Eine Beschreibung der Herleitung und Regionalisierung des BFI kann KLIWA (2012) entnommen werden. Die Übersichtskarte der BFI-Verteilung in den vier Bundesländern ist in Kopp et al. (2018) dargestellt.

Als meteorologische Antriebsdaten wurden Daten des Deutschen Wetterdienstes (DWD) für Niederschlag, Lufttemperatur, relative Luftfeuchte, Sonnenscheindauer und Windstärke in täglicher Auflösung verwendet. Die vom DWD bereitgestellten Stationsdaten (Tageswertreihen) wurden aufbereitet und auf Plausibilität geprüft (Gudera und Morhard 2015). Für die räumliche Interpolation der meteorologischen Daten wurde die Distanzgewichtung (Luftfeuchte, Sonnenscheindauer, Globalstrahlung), eine Kombination aus Distanzgewichten und Analyse der Höhenabhängigkeit (Temperatur) und ausschließlich Höhenabhängigkeit (Windstärke) unter Verwendung von 12 Nachbarpunkten angewandt. Weitere Informationen zum Interpolationsverfahren des Modells können Morhard (2012) entnommen werden. Als Niederschlagsdaten kamen die unkorrigierten REGNIE-Rasterdaten des DWD (Rauthe et al. 2013) zur Anwendung.

Für die Modellierung von Bodenwasserhaushalt und Grundwasserneubildung mit GWN-BW werden Daten zu Landnutzung, Höhenmodell und Bodenkarte benötigt, um physiographische Eingangsdaten abzuleiten. Durch die 
Verschneidung von Landnutzung (DLR 2005) und Bodenkarten wurden für die insgesamt $147.286 \mathrm{~km}^{2}$ große Fläche Süddeutschlands 401.728 Grundflächendatensätze erstellt. Diese sind in den Ländern Baden-Württemberg, Bayern und Rheinland-Pfalz in ihrer räumlichen Auflösung vergleichbar. In Hessen wurde durch die Verwendung der Bodenkarte BK50 ein höher aufgelöster Grundflächendatensatz generiert (vgl. Tab. 1 in Kopp et al. 2018).

Es wird darauf hingewiesen, dass die mit dem beschriebenen Verfahren ermittelte Grundwasserneubildung aus Niederschlag nur für das oberste Grundwasserstockwerk aussagekräftig ist, und, je nach den regionalen hydrogeologischen Gegebenheiten, nicht mit dem Grundwasserdargebot gleichgesetzt werden kann. Hierfür sind ggf. weitere Aspekte wie Grundwasseraustritte über schwebenden Aquiferen, Speichervermögen des jeweiligen Grundwasserleiters, Influenz und Effluenz entlang von Oberflächengewässern, laterale Grundwasserströmung (Randzustrom) sowie die Zusickerung und Aussickerung von Grundwasser aus bzw. in andere Grundwasserleiter zu berücksichtigen.

\section{Ergebnisse}

Als Referenzzeitraum wurde im Rahmen von KLIWA der 30-jährige Zeitraum 1971-2000 festgelegt. Dieser wird entsprechend für die Einordnung der Ergebnisse der Langzeitsimulation der Bodenwasserhaushaltsmodellierung herangezogen.

\section{Lufttemperatur}

Die Gebietsmitteltemperatur von Süddeutschland liegt für den Referenzzeitraum 1971-2000 bei 8,2 ${ }^{\circ} \mathrm{C}$. Aufgrund des stärker ozeanisch geprägten Klimas in den westlichen Regionen des Untersuchungsgebietes sind die Jahresmitteltemperaturen in Baden-Württemberg $\left(8,4^{\circ} \mathrm{C}\right)$, Hessen $\left(8,6^{\circ} \mathrm{C}\right)$ und Rheinland-Pfalz $\left(8,9^{\circ} \mathrm{C}\right)$ insgesamt höher als im eher kontinental geprägten Klima Bayerns $\left(7,8^{\circ} \mathrm{C}\right)$. Von 1951 bis Mitte der 1980er-Jahre unterliegt die Jahresmitteltemperatur im Vergleich zur Referenzperiode vergleichsweise geringen Schwankungen. Seit Ende der 1980erJahre ist im Vergleich zum Referenzzeitraum ein signifikanter Anstieg der mittleren jährlichen Temperatur in allen vier Ländern zu erkennen ( $>75$. Perzentil, rote Balken) (Abb. 1a). Das Trockenjahr 2018 (KLIWA 2019a; LUBW 2019) sticht mit der bisher höchsten Jahresmitteltemperatur seit 1951 deutlich hervor. Insbesondere im Frühjahr und Frühsommer sind die Temperaturen gegenüber der Referenzperiode zuletzt gestiegen (siehe Anhang Abb. 2).

\section{Niederschlag}

Die über Süddeutschland gemittelte jährliche Niederschlagssumme für den Zeitraum 1971-2000 beträgt $903 \mathrm{~mm} / \mathrm{a}$, wobei die Niederschlagssummen in Hessen $(770 \mathrm{~mm} / \mathrm{a})$ und Rheinland-Pfalz $(795 \mathrm{~mm} / \mathrm{a})$ deutlich geringer ausfallen als jene in Bayern $(939 \mathrm{~mm} / \mathrm{a})$ und Baden-Württemberg $(971 \mathrm{~mm} / \mathrm{a})$. Für den Gesamtzeitraum 1951-2019 ist kein langjähriger Trend zu erkennen, die Jahressummen sind durch eine große Variabilität geprägt. Auch lassen sich mehrjährige Nass- und Trockenzeiträume feststellen (Abb. 1b). In der jüngeren Vergangenheit sind die Niederschlagssummen im Kalenderjahr im Vergleich zur Referenzperiode bestenfalls durchschnittlich und haben sich seit dem Jahr 2003 gemittelt über Süddeutschland um $6 \%$ reduziert (Baden-Württemberg und Bayern:-6\%, Hessen: $-5 \%$ und Rheinland-Pfalz: $-8 \%$ ). Während sich die Niederschläge im hydrologischen Sommerhalbjahr im Vergleich zur Referenzperiode seit 2003 nur geringfügig verändert haben, sind besonders die grundwasserneubildungswirksamen Niederschläge im hydrologischen Winterhalbjahr im Mittel um $11 \%$ zurückgegangen (Baden-Württemberg und Bayern:-10\%, Hessen: $-12 \%$ und Rheinland-Pfalz: -13\%) (siehe Anhang Abb. 1) (KLIWA 2019a). In einzelnen Jahren, wie den Trockenjahren 2003, 2015 und 2018, verringerten sich die jährlichen Niederschlagssummen in den Ländern z.T. um fast ein Drittel. Dabei fiel über die Landesfläche gemittelt die geringste Niederschlagsmenge in Baden-Württemberg mit $703 \mathrm{~mm}$ $(-28 \%)$ und Bayern mit $687 \mathrm{~mm}(-27 \%)$ im Jahr 2003, wohingegen Rheinland-Pfalz mit $606 \mathrm{~mm}(-24 \%)$ im Jahr 2015 und Hessen mit nur 570 mm (-26\%) im Jahr 2018 am stärksten betroffen waren. Regional fielen die Abnahmen der Niederschlagssumme oftmals noch deutlicher aus: z.B. Pfälzerwald (2015: $-36 \%$ ), Nördlicher Oberrheingraben (2018: $-31 \%)$, Nördlinger Ries (2003: $-32 \%$ ) und Mainzer Becken (2003: -30\%) (Übersicht der Naturräume siehe Abb. 3.1 in KLIWA 2017).

\section{Grundwasserneubildung aus Niederschlag}

Die mittlere jährliche Grundwasserneubildung aus Niederschlag in Süddeutschland im Zeitraum 1971-2000 beträgt $175 \mathrm{~mm} / \mathrm{a}$ (Abb. 2a). Dabei variiert sie zwischen $107 \mathrm{~mm} / \mathrm{a}$ in Hessen und $207 \mathrm{~mm} / \mathrm{a}$ in Bayern. Tendenziell folgt die Entwicklung der Grundwasserneubildung von 1951-2002 der Entwicklung des Niederschlags und ist somit durch eine große Variabilität und einen Wechsel von mehrjährigen Nass- und Trockenperioden gekennzeichnet, wobei die Grundwasserspeicher überwiegend während der neubildungsreichen Nassjahre (Ende 1960er-Jahre, Ende 1970erJahre, Mitte 1980er und Ende 1990er-Jahre) aufgefüllt wurden. Auffallend ist der Zeitraum seit dem Trockenjahr 2003, 
Abb. 1 a Jährliche Lufttemperatur und $\mathbf{b}$ jährliche Niederschlagssumme in Süddeutschland im Zeitraum 1951 bis 2019. Die 10-Jahresmittel bzw. das 9-Jahresmittel zwischen 2011 und 2019 sind in kursiv dargestellt

Fig. 1 a Annual air temperature and $\mathbf{b}$ annual precipitation in southern Germany in the period 1951 to 2019. The 10-year averages or the 9-year average between 2011 and 2019 are presented in italics

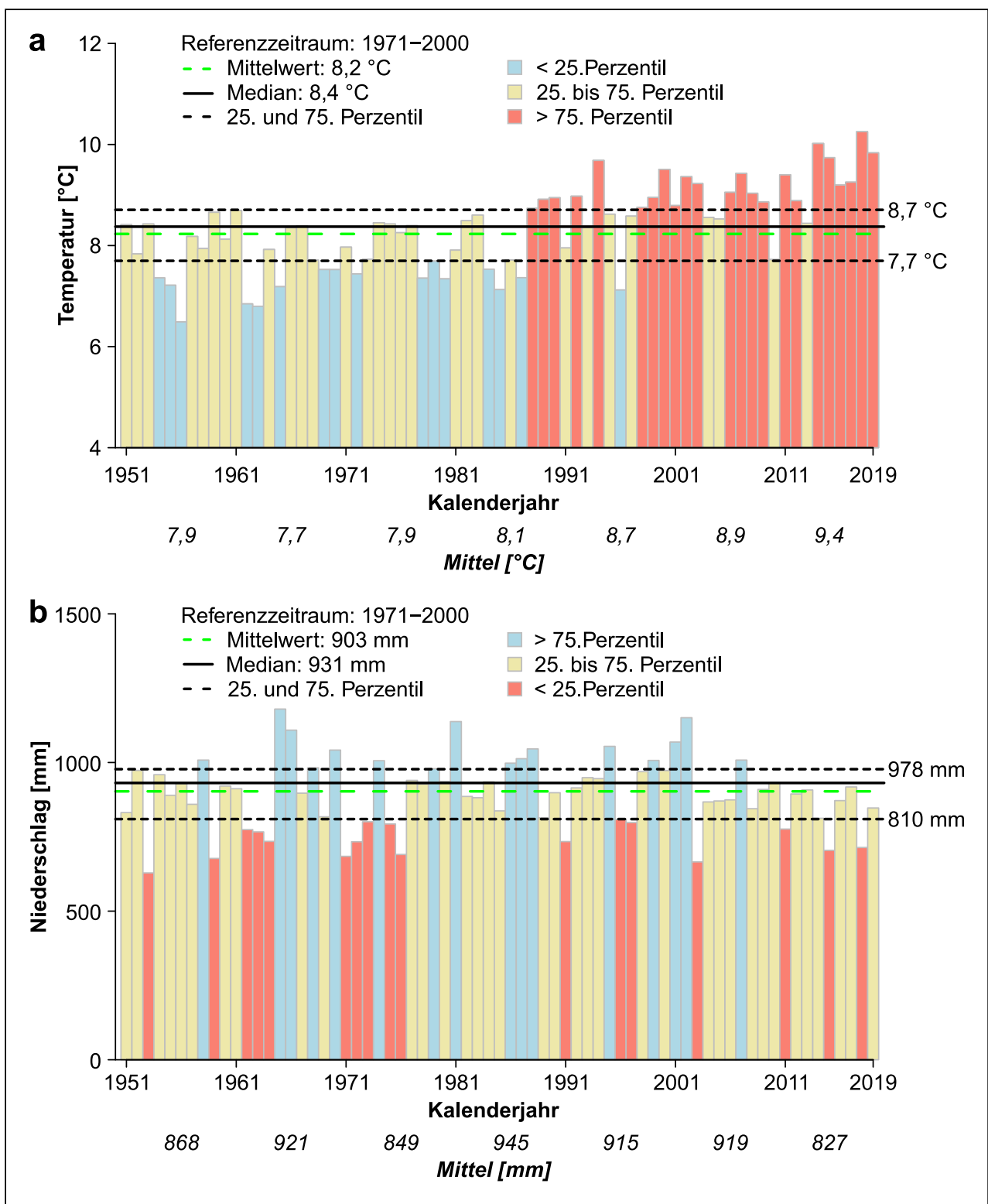

in dem, bedingt durch geringe Winterniederschläge (siehe Anhang Abb. 1) und vergleichsweise hohe Lufttemperaturen (siehe Anhang Abb. 2), nur noch unterdurchschnittliche bis maximal durchschnittliche Grundwasserneubildungsraten registriert wurden. Nassjahre mit hohen Grundwasserneubildungsraten traten seit dem Jahr 2003 gar nicht mehr in Erscheinung, wodurch die jährliche Variabilität deutlich abgenommen hat. So reduzierte sich gegenüber dem Referenzzeitraum 1971-2000 die Grundwasserneubildung in den letzten 17 Jahren (2003-2019) in Süddeutschland um 19\% (Baden-Württemberg: $-18 \%$, Bayern: $-15 \%$, Hessen: $-26 \%$ und Rheinland-Pfalz: $-26 \%$ ), wobei insbesondere in den Trockenjahren 2003, 2015, 2018 und 2019 im Vergleich zum langjährigen Mittel erhebliche Defizite auftraten. Die Trockenjahre der jüngeren Vergangenheit waren in den Ländern unterschiedlich stark ausgeprägt. Während das Jahr 2018 in Hessen für ein Defizit von $51 \%$ bei der Grundwasserneubildung aus Niederschlag sorgte, war das Jahr 2003 mit Abnahmen um fast 50\% in Baden-Württemberg und Bayern deutlich neubildungsärmer. In RheinlandPfalz hingegen waren die Abnahmen der Grundwasserneubildung im Jahr 2015 mit $-42 \%$ am höchsten. In einzelnen Naturräumen wie dem Nördlichen Oberrheingraben oder dem Mainzer Becker sanken die Grundwasserneubildungsraten im Jahr 2019 hingegen um rund $80 \%$ im Vergleich zur Referenzperiode (Übersicht der Naturräume siehe Abb. 3.1 in KLIWA 2017). 
Abb. 2 a Jährliche Grundwasserneubildung aus Niederschlag und $\mathbf{b}$ jährlicher Trockenheitsindex in Süddeutschland im Zeitraum 1951 bis 2019. Die 10Jahresmittel bzw. das 9-Jahresmittel zwischen 2011 und 2019 sind in kursiv dargestellt Fig. 2 a Annual groundwater recharge and $\mathbf{b}$ annual drought index in southern Germany in the period 1951 to 2019. The 10 -year averages or the 9-year average between 2011 and 2019 are presented in italics

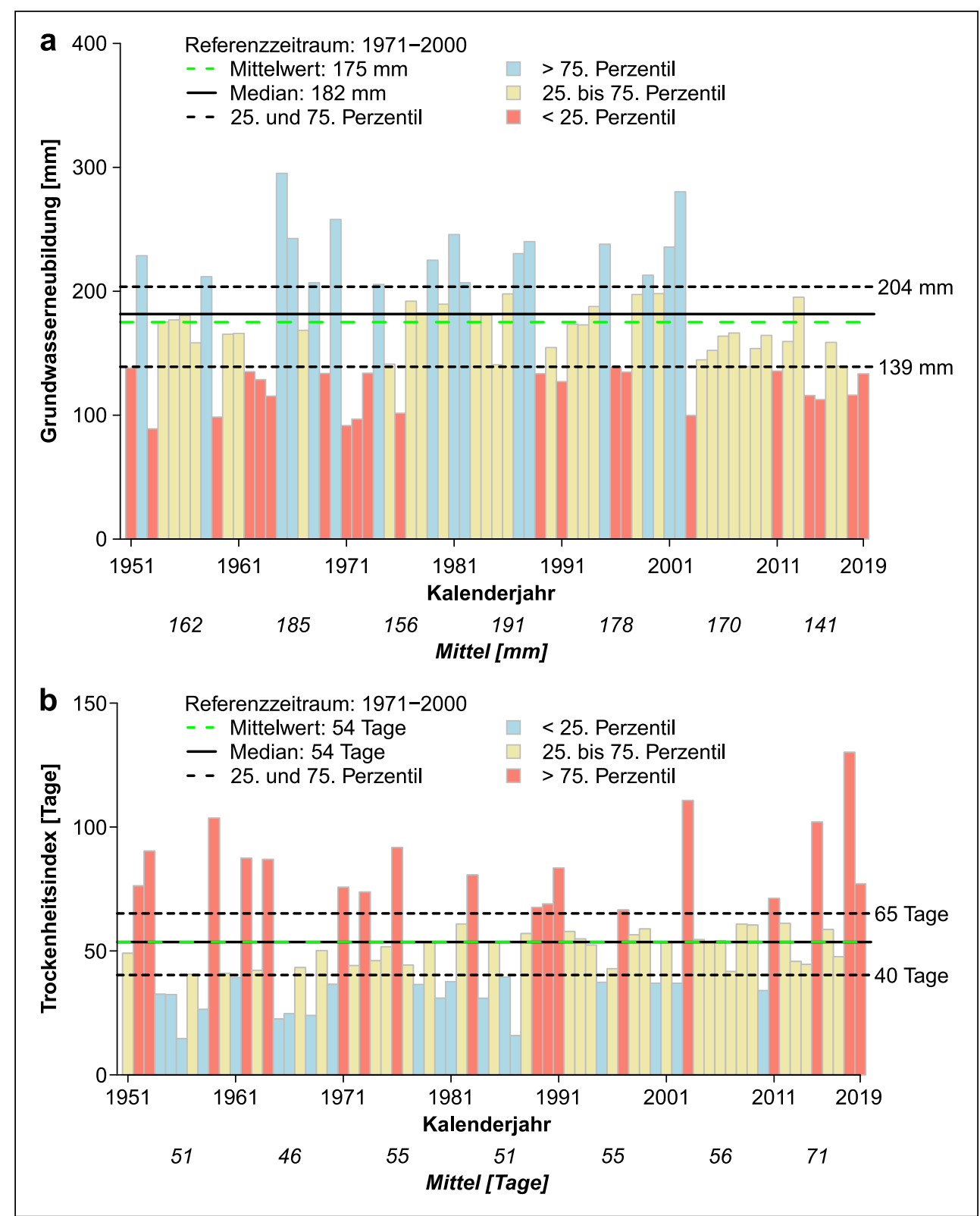

\section{Trockenheitsindex}

Der Trockenheitsindex ist definiert als die Anzahl an Tagen, an denen die Füllung des Bodenwasserspeichers 30\% der nFK unterschreitet (WaBoA 2012). Er kann somit als Maß zur Entwicklung des Trockenstresses der Vegetation herangezogen werden. Im langjährigen Mittel 1971- 2000 beträgt der Trockenheitsindex in Süddeutschland 54 Tage/ Jahr (Abb. 2b). In den Jahren 1951 bis 1990 kann kein eindeutiger Trend in der Entwicklung festgestellt werden, da sich Jahre mit einem über- und unterdurchschnittlichen Trockenheitsindex abwechseln. Seit dem Beginn der 1990erJahre ist jedoch ein leichter Anstieg des Trockenheitsindex in allen vier Bundesländern zu erkennen. Die Häufung von Trockenjahren, als Folge der zunehmenden Temperatur und der daraus resultierenden vegetations- und strahlungsbedingt steigenden Verdunstungsverluste verbunden mit geringen Niederschlägen, führt zu einer raschen Entleerung des Bodenspeichers (KLIWA 2017). Im Jahresverlauf sind in allen Bundesländern die Sommermonate am trockensten, speziell im Frühjahr zeichnet sich jedoch seit 2003 eine Zunahme des Trockenheitsindex ab (siehe Anhang Abb. 3). Der bislang mit Abstand höchste Wert des Trockenheitsindex seit 1951 tritt, gemittelt über alle 4 Länder, im Jahr 2018 mit 130 Tagen auf (Baden-Württemberg: 130 Tage, Bayern: 116 Tage, Hessen: 152 Tage und Rheinland-Pfalz: 157 Tage). Der bisherige Höchstwert für Süddeutschland aus dem Jahr 2003 mit 111 Tagen wurde deutlich übertroffen (Ba- 
den-Württemberg: 107 Tage, Bayern: 107 Tage, Hessen: 115 Tage und Rheinland-Pfalz: 127 Tage). Auffällig beim Trockenheitsindex ist die hohe Variabilität der Jahreswerte zwischen 1951 und 2019, was als Hinweis auf eine besondere Sensitivität gegenüber meteorologischen Änderungen und somit langfristig auch dem Klimawandel interpretiert werden kann.

\section{Grundwasserstände und Quellschüttungen}

Um die Grundwasserstände und Quellschüttungen kontinuierlich überwachen und bewerten zu können, betreibt die Wasserwirtschaftsverwaltung der einzelnen Bundesländer flächendeckende Messnetze aus repräsentativen Messstellen. Auswertungen zum Langzeitverhalten der erhobenen Grundwasserstände und Quellschüttungen an ausgewählten Messstellen in Süddeutschland bis zum Jahr 2007 (KLIWA 2011) und bis zum Jahr 2015 (LfU 2016) ${ }^{1}$ zeigen langfristig eine Tendenz zur Verringerung der mittleren Grundwasserstände und Quellschüttungen. In den letzten Jahren hat sich diese Entwicklung weiter fortgesetzt und zeigt damit die direkten Auswirkungen des Rückgangs der Grundwasserneubildung auf.

Eine gemeinsame länderübergreifende Auswertung zum Auftreten von Niedrigstwerten im Zeitraum 1971 bis 2019 (Datengrundlage: 897 Grundwasser- und Quellmessstellen)

\footnotetext{
${ }^{1}$ Bayerisches Landesamt für Umwelt (LfU): Langzeitverhalten von Grundwasserständen und Quellschüttungen in Baden-Württemberg, Bayern, Hessen und Rheinland-Pfalz. Unveröffentlichter Bericht. IAWG. Ottobrunn (2016)
}

zeigt die sehr niedrigen Grundwasserstände in den 1970erJahren, die auch in einem Großteil Deutschlands zur bisher schwersten „Grundwasserdürre“ führten (Hellwig 2019). Eine vergleichbare Dürresituation wiederholte sich mit einer ausgeprägten Häufung von Niedrigstwerten in den Jahren 2018 und 2019 in Süddeutschland (Abb. 3). Dies ist eine Konsequenz aus mehrjährigen Zeiträumen, in denen durch geringe Niederschlagsmengen und zum Teil auch hohe Temperaturen (Abb. 1) nur wenig Grundwasserneubildung aus Niederschlag erfolgte (Abb. 2b).

Die Anzahl der hier ausgewerteten Messstellen der Bundesländer, die kontinuierlich seit 1971 im Messbetrieb sind, variiert von 65 Messstellen in Bayern bis zu 365 Messstellen in Hessen (Baden-Württemberg: 152 Messstellen, Rheinland-Pfalz: 315 Messstellen). Während die größte Anzahl an Messstellen mit Niedrigstwerten in Hessen in den 1970er-Jahren verzeichnet wurde, wurden in Baden-Württemberg, Bayern und Rheinland-Pfalz in den Jahren 2018 und 2019 die meisten Niedrigstwerte an den Messstellen registriert (siehe Anhang Tab. 1).

\section{Trockenwetterdargebot}

Die Auswertung der Langzeitsimulation mit dem Modell GWN-BW von 1951 bis 2019 zeigt anschaulich die langfristige Entwicklung der Wasserhaushaltsgrößen in Abhängigkeit der meteorologischen Randbedingungen in Süddeutschland (Abb. 1). Besonders der Anstieg der Lufttemperatur seit Ende der 1980er-Jahre und die Veränderungen der jährlichen Niederschlagssummen wirken sich auf den sensitiven Wasserbilanzrest Grundwasserneubil-
Abb. 3 Eintrittsjahr des Niedrigstwertes an 897 repräsentativen Grundwassermessstellen und Quellen in Süddeutschland im Zeitraum 1971-2019

Fig. 3 Year of entry of the lowest recorded water level at 897 representative groundwater monitoring stations and springs in southern Germany within the period 1971-2019

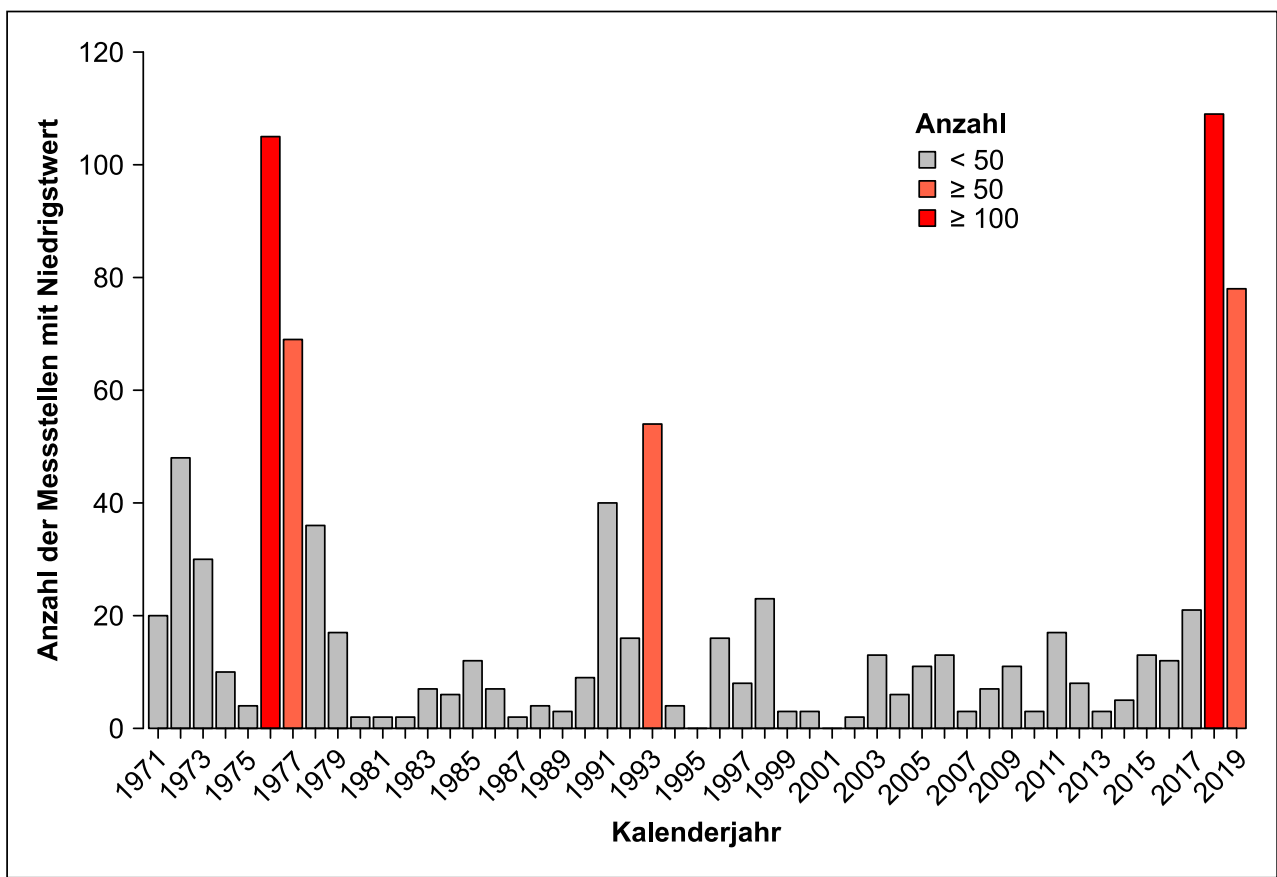


dung aus Niederschlag aus (Abb. 2a). Eine Verminderung der Grundwasserneubildung in niederschlagsarmen Mehrjahreszeiträumen bewirkt fallende Grundwasserstände und abnehmende Quellschüttungen und kann im Grundwasser zu einer ausgeprägten Niedrigwassersituation führen.

Eine nachhaltige Bewirtschaftung von Grundwasservorkommen stützt sich auf das „nutzbare Grundwasserdargebot" (nach DIN 1994), das unter anderem von der Grundwasserneubildung, dem Speichervermögen des Untergrundes und weiteren Randbedingungen wie Gewinnbarkeit, Grundwasserbeschaffenheit oder Ökologie abhängt.

In der Praxis werden zur Abschätzung des ,nutzbaren Grundwasserdargebots“ im Rahmen von wasserwirtschaftlichen Fragestellungen in der Regel mittlere langjährige Grundwasserneubildungsraten (30 Jahre) herangezogen. Diese überschätzen aber unter sich wandelnden Klimabedingungen für mehrjährige Trockenperioden die realen Bedingungen, was in einer Übernutzung des Dargebots resultieren kann. Speziell die jüngste Entwicklung seit 2003 führte zu einer Änderung einzelner Wasserbilanzgrößen, die sich von den langjährigen Verhältnissen (1971-2000) teils erheblich unterscheiden. Wie anhand der modellierten Grundwasserneubildung aus Niederschlag und den gemessenen Grundwasserständen und Quellschüttungen anschaulich gezeigt werden kann, traten seit 2003 rückläufige Tendenzen auf, welche sich in den letzten Jahren weiter verstärkten.

Aus diesem Grund wurde in KLIWA eine Handlungsempfehlung entwickelt, welche im Sinne des Vorsorgegedankens empfiehlt, für wasserrechtliche Verfahren definierte Trockenzeiträume von 10 Jahren zugrunde zu legen (KLIWA 2017). Dies ist auch aus wasserrechtlicher Sicht gegeben. So ist nach $\S 6$ des Wasserhaushaltsgesetzes den möglichen Folgen des Klimawandels im Zusammenhang mit der Gewässerbewirtschaftung vorzubeugen (Dohmen 2020).

Auswertungen von gleitenden 10-Jahreszeiträumen (Dekaden) der Grundwasserneubildung aus Niederschlag glätten die jährlichen Schwankungen und zeigen den längerfristig zugrunde liegenden Trend. Während es in den 1970erJahren mehrere aufeinanderfolgende neubildungsarme Jah- re gab, wurden die Grundwasservorräte in den 1980erJahren und zum Jahrtausendwechsel (1999-2002) wieder aufgefüllt. Seitdem sind die Werte insgesamt rückläufig und befinden sich aktuell deutlich unterhalb des Niveaus der 1970er-Jahre. Um diese mehrjährige Entwicklung darstellen zu können, werden in Abb. 4 die 10-jährigen gleitenden Mittelwerte der Grundwasserneubildung aus Niederschlag für die Bundesländer, normiert auf die Referenzperiode 1971 bis 2000, dargestellt. Dabei sind Zeiträume mit einer überdurchschnittlichen Grundwasserneubildung durch Werte $>100 \%$ und Zeiträume mit unterdurchschnittlicher Grundwasserneubildung durch Werte $<100 \%$ besonders deutlich zu erkennen. In allen vier Bundesländern hat sich in den letzten Jahren ein Grundwasserneubildungsdefizit aufgebaut, was einen zunehmenden, auch überregionalen, Handlungsbedarf aufzeigt. Die Durchführung von KLIWAStresstests mit einem 30-jährigen synthetischen Szenario auf Basis von gemessenen (Extrem-) Jahren (KLIWA 2019b) in regionalen Fallstudiengebieten in Bayern haben gezeigt, dass je nach Speicherfähigkeit des Untergrunds erst mehrere Nassjahre (Niederschlag min. $+15 \%$ vom langjährigen Mittel) in Folge zu einer vollständigen Entspannung der Situation bzw. zum Auffüllen der Grundwasserspeicher führen. Dies zeigt sich insbesondere an Quellschüttungen oberflächennaher Aquifere, da diese Quellen besonders sensitiv auf Änderungen des Klimageschehens reagieren.

Um die Regionen innerhalb der einzelnen Bundesländer identifizieren zu können, welche durch die aktuelle Trockenperiode am stärksten betroffen sind, erfolgte eine Auswertung der gleitenden 10-Jahres-Zeiträume für jede Grundfläche des insgesamt 401.728 Einzelflächen umfassenden Grundflächendatensatzes der Bodenwasserhaushaltsmodellierung. Als Ergebnis lässt sich die Eintrittszeitspanne der jeweiligen Dekade mit der geringsten Grundwasserneubildung (Trockendekade) innerhalb des Gesamtzeitraumes 1951-2019 in hoher räumlicher Auflösung ableiten.

In Tab. 1 sind die fünf Trockendekaden mit der größten räumlichen Ausbreitung in Süddeutschland gelistet (Auszug aus Tab. 2 im Anhang). Besonders deutlich wird hier die große räumliche Ausbreitung der aktuellen Dekade

Tab. 1 Auflistung der fünf Dekaden mit der geringsten Grundwasserneubildung in Süddeutschland. Enthalten ist die jeweilige Gesamtzahl an betroffenen Grundflächen, die jeweils betroffene Fläche sowie der Flächenanteil an der Gesamtfläche von $147.286 \mathrm{~km}^{2}$

Table 1 List of the five decades with the lowest groundwater recharge in southern Germany with the number of affected individual areas, the area and the percentage of the affected area in relation to the total area of $147,286 \mathrm{~km}^{2}$

\begin{tabular}{llll}
\hline Dekade & Anzahl der Grundflächen & $\begin{array}{l}\text { Fläche } \\
{\left[\mathrm{km}^{2}\right]}\end{array}$ & $1 \%]$ \\
\hline $1955-1964$ & & 19.598 & 13 \\
$1969-1978$ & 32.911 & 7945 & 5 \\
$1971-1980$ & 35.298 & 10.458 & 7 \\
$2003-2012$ & 36.473 & 21.820 & 15 \\
$2010-2019$ & 57.174 & 39.550 & 27 \\
\hline
\end{tabular}


2010-2019, insbesondere in Südostbayern und Hessen, sowie in Teilen von Baden-Württemberg und RheinlandPfalz (Abb. 5a).

Analog zur Grundwasserneubildung sind in Tab. 2 die fünf Dekaden mit der jeweils höchsten Anzahl an Tagen mit Trockenstress (Trockenheitsindex) in Süddeutschland gelistet (Auszug aus Tab. 2 im Anhang). Besonders auffallend ist hier, auch im Vergleich zur Grundwasserneubildung, die deutliche räumliche Dominanz der aktuellen Dekade 2010-2019 in allen untersuchten Bundesländern (Abb. 5b).

Gründe hierfür sind die unterdurchschnittlichen Niederschlagssummen der letzten Jahre bei einem gleichzeitigen Anstieg der Jahresmitteltemperatur. Das durch insgesamt höhere Temperaturen begünstigte Vegetationswachstum und die verlängerten Vegetationsperioden führten zu einer Zunahme der Transpiration und somit zu einer Abnahme des pflanzenverfügbaren Wassers im Boden. In den Dekaden 2009-2018 bzw. 2010-2019 wurde als Folge der letzten Trockenjahre mehrfach in weiten Teilen Süddeutschlands eine weitgehende und tiefgründige Entleerung des Bodenwasserspeichers ( $<30 \% \mathrm{nFK})$ beobachtet (Marx 2020; Marx et al. 2016; Hellwig 2019).

Die beschriebenen Unterschiede zwischen Grundwasserneubildung und Trockenheitsindex (Abb. 5) sind zum Teil auch Folge der unterschiedlichen zeitlichen Dynamik der relevanten Einzelprozesse. Während der Trockenheitsindex unmittelbar auf eine Entleerung des Bodenwasserspeichers reagiert, erfolgt eine Auffüllung der Grundwasservorräte erst zeitlich verzögert durch die Bildung von Sickerwasser und nach weitgehender Sättigung des Bodens. Eine nachhaltige Regeneration der Grundwasservorkommen kann daher oft erst im Verlauf des anschließenden Winterhalbjahres stattfinden. Besonders anschaulich war dies im Winterhalbjahr 2018/2019 zu beobachten, als regional erst ab Februar unter weitgehend wassergesättigten Bedingungen wieder nennenswerte Sickerwasserbildung beziehungsweise Grundwasserneubildung einsetzte (Raspe et al. 2020). Der Trockenheitsindex als vergleichsweise schnell reagierender Indikator kann damit auch als ein frühzeitiges Anzeichen für ausbleibende Grundwasserneubildung herangezogen werden.

\section{Diskussion, Schlussfolgerung und Ausblick}

Grundwasser ist sowohl elementare Lebensgrundlage als auch Wirtschaftsgut. Die Sicherung der Lebensgrundlage Grundwasser stellt im Kontext des Klimawandels auch eine globale Gemeinschaftsaufgabe dar (Watzel 2020). In Deutschland ist Grundwasser ein Schlüsselelement der Wasserversorgung, insbesondere der Trinkwasserversor-

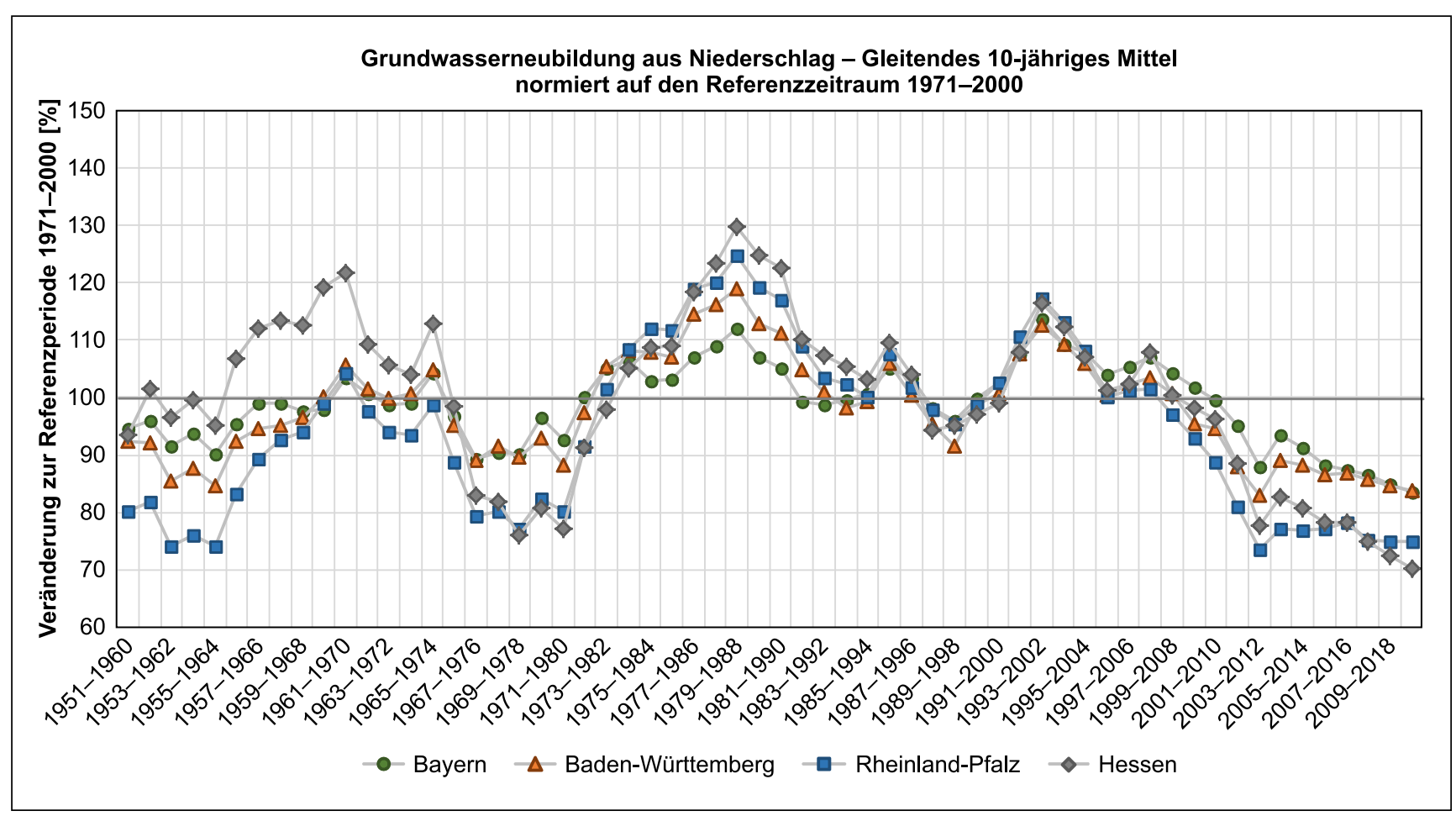

Abb. 4 Gleitendes 10-jähriges Mittel der Grundwasserneubildung aus Niederschlag der Bundesländer Baden-Württemberg, Bayern, Hessen und Rheinland-Pfalz im Zeitraum 1951-2019. Die Mittelwerte sind auf den Referenzzeitraum 1971-2000 normiert

Fig. 4 Moving 10-year average of groundwater recharge from precipitation in the federal states of Baden-Wuerttemberg, Bavaria, Hesse and Rhineland-Palatinate within the period 1951-2019. The relative deviations are based on the mean value 1971-2000 (reference period) 


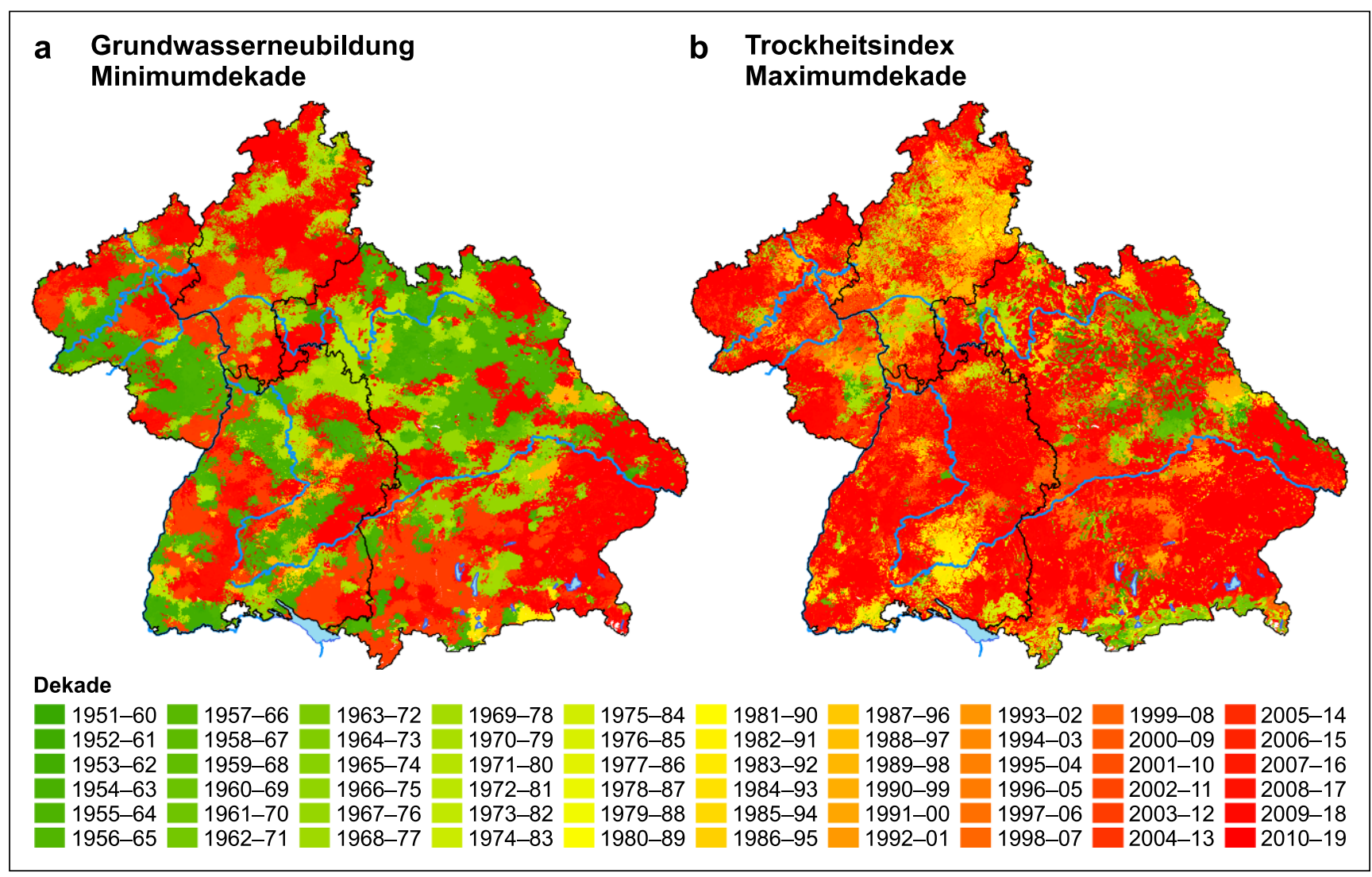

Abb. 5 Eintrittszeitspanne (Dekade) der niedrigsten mittleren 10-jährigen Grundwasserneubildung (a) und des höchsten mittleren Trockenheitsindex (b) je Grundfläche, aufgetreten im Zeitraum 1951-2019

Fig. 5 Entry period (decade) with the lowest mean groundwater recharge (a) and with the highest drought index (b) for each individual model area within the period 1951-2019

Tab. 2 Auflistung der fünf Dekaden mit der höchsten Anzahl an Tagen mit Trockenstress (=Trockenheitsindex) in Süddeutschland. Enthalten ist die jeweilige Gesamtzahl an betroffenen Grundflächen, die jeweils betroffene Fläche sowie der Flächenanteil an der Gesamtfläche von $147.286 \mathrm{~km}^{2}$

Table 2 List of the five decades with the highest number of days with drought index in southern Germany with the number of affected individual areas, the area and the percentage of the affected area in relation to the total area of $147,286 \mathrm{~km}^{2}$

\begin{tabular}{llll}
\hline Dekade & Anzahl der Grundflächen & $\begin{array}{l}\text { Fläche } \\
{\left[\mathrm{km}^{2}\right]}\end{array}$ & {$[\%]$} \\
\hline $1976-1985$ & 15.353 & 3699 & 3 \\
$1988-1997$ & 52.359 & 8543 & 6 \\
$2003-2012$ & 19.750 & 7448 & 5 \\
$2009-2018$ & 32.483 & 14.360 & 10 \\
$2010-2019$ & 177.888 & 70.743 & 48 \\
\hline
\end{tabular}

gung. Besonders die Jahre 2018 und 2019 haben eindrücklich gezeigt, dass es während länger andauernder Trockenheit auch in einem vermeintlich wasserreichen Land wie Deutschland örtlich und zeitlich begrenzt zu Wasserknappheit kommen kann (DVGW 2020). Die erkennbare erhebliche Zunahme des Trockenheitsindex seit dem Jahrtausendwechsel (Tab. 2, Abb. 2b, siehe Anhang
Abb. 3) verdeutlicht dies anschaulich und zeigt auch einen zunehmenden Nutzungskonflikt auf.

Um Ertragsrückgänge zu vermeiden, führte die zunehmende Trockenheit zu einem steigenden Bewässerungsbedarf in der Landwirtschaft. So stieg alleine im Zeitraum 2010 bis 2015 der Anteil an bewässerter landwirtschaftlicher Fläche in Deutschland um rund 6\% (Thober et al. 2018; Anter et al. 2018). Gleichzeitig erreichte die Grundwasserneubildung im selben Zeitraum in vielen Regionen Süddeutschlands ein Minimum (Abb. 4 und 5, Tab. 1), aufgrund von zumeist unterdurchschnittlichen bis maximal durchschnittlichen Winterniederschlägen (siehe Anhang Abb. 1). Diese wiederum führten zu sinkenden Grundwasserständen (Abb. 3) und bei einem zum Teil stark ansteigenden Spitzenwasserbedarf zu vorübergehenden lokalen Engpässen in der Trinkwasserversorgung.

In Anbetracht der Tatsache, dass in Süddeutschland ein Großteil der Trinkwasserversorgung auf der Nutzung von Grund- und Quellwasser beruht, müssen umfangreiche und transparente Handlungs- und Anpassungskonzepte entwickelt werden, um absehbaren Konflikten vorzubeugen oder sie zumindest zu minimieren (BMU 2015; KLIWA 2018). Auch wenn laut DVGW die Trinkwasserversorgung 
in Deutschland durch die jüngsten Trockenperioden nicht gefährdet war, ist die Wasserversorgungsinfrastruktur in einigen Kommunen an ihre Grenzen gestoßen (DVGW 2020; Deutscher Bundestag 2020). Infolge des durch die Trockenheit der letzten Jahre gestiegenen Nutzungsdrucks hat Bundesumweltministerin Svenja Schulze im August 2020 ein Regelkonzept für den Umgang mit Wasser angekündigt. Durch eine nationale Wasserstrategie soll eine Hierarchie für die Wassernutzungen festgelegt und ein bundesweites Wassermanagement ermöglicht werden. Bei lokalen Engpässen in der Wasserversorgung sollen somit zukünftig klare Regeln gelten, welche Nutzung Priorität hat. Die Veröffentlichung des Entwurfs der Wasserstrategie ist für Juni 2021 geplant (BMU 2020).

Die Notwendigkeit einer Wasserstrategie zeigt sich umso mehr vor dem Hintergrund der voranschreitenden Klimaerwärmung, welche voraussichtlich mit einer weiteren Verschärfung der bereits stattfindenden Entwicklung (mehr Starkregen, weniger Schneerücklage, mehr potenzielle Verdunstung) bei gleichzeitig zunehmenden Wasserbedarf (Spitzenwasserbedarf, Bewässerungsbedarf) einhergeht (KLIWA 2019a). In der Folge wird zukünftig das Auftreten einer regionalen temporären Wasserknappheit wahrscheinlicher. Diese ist jedoch zusätzlich auch in hohem Maße von der Entwicklung des Pro-Kopf-Verbrauchs und der jeweiligen regionalen demographischen Entwicklung abhängig (LfU online 2020).

Erste Berechnungen mit regionalen Klimaprojektionen lassen eine flächenhafte Verringerung der Grundwasserneubildung aus Niederschlag in der nahen und fernen Zukunft erwarten (KLIWA 2012, 2017). So ist es wahrscheinlich, dass die zum Teil projizierten feuchteren Winter und insgesamt trockeneren Sommer die Grundwasserneubildung und die Grundwasserstände größeren saisonalen Schwankungen unterwerfen werden. $\mathrm{Zu}$ beachten ist, dass die seit 2003 beobachteten reduzierten Niederschläge im hydrologischen Winterhalbjahr (siehe Anhang Abb. 1) hierzu scheinbar im Widerspruch stehen. Inwieweit dies einen durch den Klimawandel bedingten langfristigen Trend darstellt, ist derzeit aber noch unklar, da der Betrachtungszeitraum von 17 Jahren aus Sicht der Klimaforschung für Langzeitaussagen noch zu kurz ist. Die hingegen bereits zu beobachtende und zukünftig projizierte Abnahme der Sommerniederschläge führt zusätzlich zu einer Abnahme der Quellschüttungen in den Mittelgebirgsregionen während der Sommerund Herbstmonate. Dadurch ist vor allem die auf Quellwasser beruhende dezentrale Trinkwasserversorgung während dieser Zeiten zunehmend gefährdet (LfU online 2020). Die Zahl der bisher betrachteten Klimaprojektionen im Grundwasserbereich ist jedoch noch zu gering, um allein auf dieser Basis eine abgesicherte Bandbreite für eine mögliche künftige Entwicklung relevanter Wasserhaushaltsgrößen aufzuspannen. Daher werden aktuell weitere regiona- le Klimaprojektionen als Grundlage zur Abschätzung der zukünftigen Entwicklung relevanter Wasserhaushaltsgrößen gerechnet. Erste Ergebnisse für Süddeutschland liegen bereits vor.

Um der weiteren Entwicklung vorzubeugen, wird daher als Alternative zu langjährigen Mittelwerten die Verwendung eines „Trockenwetterdargebots“ als Grundlage wasserrechtlicher Bewirtschaftungsentscheidungen vorgeschlagen. Mit diesem Ansatz, bzw. einer entsprechend niedrigeren Grundwasserneubildung auf Basis eines zehnjährigen Trockenzeitraums, sollen in Zukunft die Trinkwasserversorgung sowie alle weiteren Nutzungen der Ressource Grundwasser auch während Trockenphasen langfristig und nachhaltig sichergestellt werden. Die Verwendung des Trockenwetterdargebots dient somit als „Klimaabschlag“ zur Abschätzung von klimawandelbedingten Änderungen des nutzbaren Grundwasserdargebots und bietet eine Empfehlung für Behörden, ihre wasserrechtlichen Bewirtschaftungsentscheidungen im Kontext der voraussichtlich häufigeren und intensiveren Niedrigwassersituationen zu begründen (Dohmen 2020).

Als Grundlage für die Ableitung des Trockenwetterdargebots dienen die länderübergreifenden Ergebnisse der Langzeitsimulation mit dem Modell GWN-BW. Die bisher vorliegenden Ergebnisse finden in Bayern, BadenWürttemberg, Hessen und Rheinland-Pfalz bereits Eingang in die wasserwirtschaftliche Planung (Fortschreibung der Wasserversorgungsbilanzen in den Regierungsbezirken Bayerns, Masterplan Wasserversorgung Baden-Württemberg, wasserwirtschaftlicher Fachplan und Leitbildprozess „Integriertes Wasserressourcen-Management Rhein-Main“ in Hessen und „Ermittlung der Vulnerabilität und Entwicklung von Anpassungsstrategien zur Sicherstellung der Wasserversorgung“ in Rheinland-Pfalz).

Funding Open Access funding enabled and organized by Projekt DEAL.

Open Access Dieser Artikel wird unter der Creative Commons Namensnennung 4.0 International Lizenz veröffentlicht, welche die Nutzung, Vervielfältigung, Bearbeitung, Verbreitung und Wiedergabe in jeglichem Medium und Format erlaubt, sofern Sie den/die ursprünglichen Autor(en) und die Quelle ordnungsgemäß nennen, einen Link zur Creative Commons Lizenz beifügen und angeben, ob Änderungen vorgenommen wurden.

Die in diesem Artikel enthaltenen Bilder und sonstiges Drittmaterial unterliegen ebenfalls der genannten Creative Commons Lizenz, sofern sich aus der Abbildungslegende nichts anderes ergibt. Sofern das betreffende Material nicht unter der genannten Creative Commons Lizenz steht und die betreffende Handlung nicht nach gesetzlichen Vorschriften erlaubt ist, ist für die oben aufgeführten Weiterverwendungen des Materials die Einwilligung des jeweiligen Rechteinhabers einzuholen.

Weitere Details zur Lizenz entnehmen Sie bitte der Lizenzinformation auf http://creativecommons.org/licenses/by/4.0/deed.de. 


\section{Literatur}

Ad-hoc-Arbeitsgruppe Boden: Bodenkundliche Kartieranleitung KA5. 438 S.; E. Schweizerbart'sche Verlagsbuchhandlung, Hannover (2005)

Anter, A., Kreins, P., Heidecke, C., Gömann, H.: Entwicklung des regionalen Bewässerungsbedarfs-Engpässe in der Zukunft? Bewässerung in der Landwirtschaft. Tagungsband zur Fachtagung, Suderburg, 11.-12.09.2017.136 S. Braunschweig (2018)

Armbruster, V.: Grundwasserneubildung in Baden-Württemberg. Freiburger Schriften zur Hydrologie 17. Institut für Hydrologie der Universität, Freiburg i. Br (2002)

Bayerisches Landesamt für Umwelt (LfU): Niedrigwasser in Bayern Grundlagen, Veränderung und Auswirkungen. 240 S.; LfU, Augsburg (2017)

Bayerisches Landesamt für Umwelt: Gewässerkundlicher Jahresbericht 2018. LfU, Augsburg (2019)

Bayerisches Landesamt für Umwelt: Wasserversorgungsbilanzen der Regierungsbezirke Unterfranken, Mittelfranken, Oberfranken, Oberpfalz, Schwaben, Niederbayern, Oberbayern (2020). https:// www.lfu.bayern.de/wasser/trinkwasserversorgung_oeffentlich/ projekte/index.htm Zugegriffen: 15.05.2020

Bergström, S.: Development and application of a conceptual runoff model for Scandinavian catchments. 134 S.; SMHI Report RHO 7. Norrköping (1976)

Bund/Länder-Arbeitsgemeinschaft Wasser (LAWA):: Auswirkungen des Klimawandels auf die Wasserwirtschaft. Bestandsaufnahme, Handlungsoptionen und strategische Handlungsfelder (2017)

Bundesministerium für Umwelt, Naturschutz und nukleare Sicherheit (BMU): Fortschrittsbericht zur Deutschen Anpassungsstrategie an den Klimawandel (2015). https://www.bmu.de/fileadmin/ Daten_BMU/Download_PDF/Klimaschutz/klimawandel_das_ fortschrittsbericht_bf.pdf Zugegriffen: 15.12.2020

Bundesministerium für Umwelt, Naturschutz und nukleare Sicherheit (BMU): Nationaler Wasserdialog (2020). https://www.bmu.de/ wasserdialog/\#c32466. Zugegriffen: 15.12.2020

Deutscher Bundestag: Antwort auf die Kleine Anfrage der Abgeordneten Bettina Hoffmann, Steffi Lemke, Lisa Badum, weiterer Abgeordneter und der Fraktion BÜNDNIS 90/DIE GRÜNEN - Drucksache 19/19265 - Entwicklung der Bodenfeuchtigkeit in Deutschland (2020). https://polit-x.de/de/documents/3803113/

Deutscher Wetterdienst: Klimastatusbericht Deutschland Jahr 2019. 23 S.; DWD, Geschäftsbereich Klima und Umwelt, Offenbach (2020)

Deutsches Zentrum für Luft- und Raumfahrt e. V. (DLR): CORINE Land Cover 2000 - Europaweit harmonisierte Aktualisierung der Landnutzungsdaten für Deutschland. Abschlussbericht zum F+E Vorhaben UBA FKZ 20112 209. 83 S.; im Auftrag des Umweltbundesamtes, Dessau (2005)

DIN: 4049-3:1994-10. Hydrologie - Teil 3: Begriffe zur quantitativen Hydrologie (1994-10). https://doi.org/10.31030/2644617 (1994)

Dohmen, A.: Wasserrechtliche Benutzungen vor den Herausforderungen des Klimawandels. Korrespondenz Wasserwirtsch. 13(8), 436-441 (2020)

DVGW: Die Wasserversorgung im Trockenjahr 2018 - Stressindikatoren und Ergebnisse einer aktuellen DVGW-Umfrage. Energ. Wasser Prax. 10/2020, 38-42 (2020)

Gudera, T., Morhard, A.: Hoch aufgelöste Modellierung des Bodenwasserhaushalts und der Grundwasserneubildung mit GWN-BW. Hydrol. Wasserbewirtsch. 59(5), 205-216 (2015)

Hellwig, J.: Grundwasserdürren in Deutschland von 1970 bis 2018. Korrespondenz Wasserwirtsch. 12(10), 567-572 (2019)

Hessisches Landesamt für Naturschutz, Umwelt und Geologie: Gewässerkundlicher Jahresbericht 2018. Hydrologie in Hessen 18. 79 S.; HLNUG, Wiesbaden (2019)

Klimaveränderung und Wasserwirtschaft (KLIWA): Langzeitverhalten von Grundwasserständen, Quellschüttungen und grundwasserbür- tigen Abflüssen in Baden-Württemberg, Bayern und RheinlandPfalz. KLIWA-Berichte, Heft 16. (2011)

Klimaveränderung und Wasserwirtschaft (KLIWA): Auswirkungen des Klimawandels auf Bodenwasserhaushalt und Grundwasserneubildung in Baden-Württemberg, Bayern und RheinlandPfalz. Untersuchungen auf Grundlage von WETTREG2003- und WETTREG2006-Klimaszenarien. KLIWA-Berichte, Heft 17. (2012)

Klimaveränderung und Wasserwirtschaft (KLIWA): Entwicklung von Bodenwasserhaushalt und Grundwasserneubildung in BadenWürttemberg, Bayern, Rheinland-Pfalz und Hessen (1951-2015). KLIWA-Berichte, Heft 21. (2017)

Klimaveränderung und Wasserwirtschaft (KLIWA): Niedrigwasser in Süddeutschland - Analysen, Szenarien und Handlungsempfehlungen. KLIWA-Berichte, Heft 23. (2018)

Klimaveränderung und Wasserwirtschaft (KLIWA): Das Jahr 2018 im Zeichen des Klimawandels - Viel Wärme, wenig Wasser in Süddeutschland. 14 S. KLIWA-Kurzbericht. (2019a)

Klimaveränderung und Wasserwirtschaft (KLIWA): Leitfaden zur Durchführung von KLIWA-Stresstests. 19 S. KLIWA-Kurzbericht. (2019b)

Kopp, B., Baumeister, C., Gudera, T., Hergesell, M., Kampf, J., Morhard, A., Neumann, J.: Entwicklung von Bodenwasserhaushalt und Grundwasserneubildung in Baden-Württemberg, Bayern, Rheinland-Pfalz und Hessen von 1951 bis 2015. HyWa 62(2), 62-76 (2018)

Landesamt für Umwelt Rheinland-Pfalz: Der Sommer 2018 in Rheinland-Pfalz. Ein wasserwirtschaftlicher Bericht. 61S.; LfU RP, Mainz (2019)

Landesanstalt für Umwelt Baden-Württemberg: Zu warm, zu heiß, zu trocken? Eine klimatische Einordnung des Jahres 2018 für BadenWürttemberg. 28 S.; LUBW, Karlsruhe (2019)

Marx, A., Samaniego, L., Kumar, R., Thober, S., Mai, J., Zink, M.: Der Dürremonitor - Aktuelle Informationen zur Bodenfeuchte in Deutschland. Forum Hydrol. Wasserbewirtsch. 37(16), 131-142 (2016)

Marx, A.: Dürremonitor Deutschland - Jährliche Dürrestärken in Deutschland 1952-2019. Stand 12.05.2020 (2020). https://www. ufz.de/index.php?de=47252. Zugegriffen: 15.05 .2020

Morhard, A.: Kurzbeschreibung des Modells GWN-BW - Erweiterungen in Version 2.0. Stand 13.05.2009. 25 S. (2009) https://www. hydrosconsult.com/hydrologie/bodenwasserhaushalt/modelldokugwn-bw/. Zugegriffen: 15.05.2020

Morhard, A.: Kurzbeschreibung des Modells GWN-BW. Bedienung und Erweiterungen in Version 3.x. Stand 13.08.2012. 32 S.(2012). https://www.hydrosconsult.com/hydrologie/bodenwas serhaushalt/modelldoku-gwn-bw/. Zugegriffen: 15.05.2020

IPCC: Klimaänderung 2014: Synthesebericht. Beitrag der Arbeitsgruppen I, II und III zum Fünften Sachstandsbericht des Zwischenstaatlichen Ausschusses für Klimaänderungen (IPCC) [Hauptautoren, R.K. Pachauri und L.A. Meyer (Hrsg.)]. IPCC, Genf, Schweiz. Deutsche Übersetzung durch Deutsche IPCC-Koordinierungsstelle, Bonn (2016)

Raspe, S., Foullois, N., Neumann, J., Zimmermann, L.: Wasserversorgung für Wald und Mensch. Trends und Auswirklungen von Trockenjahren am Beispiel des Hochspessarts. Wald und Wasser. LWF-aktuell 126. (2020)

Rauthe, M., Steiner, H., Riediger, U., Mazurkiewicz, A., Gratzki, A.: A Central European precipitation climatology - Part I: Generation and validation of a highresolution gridded daily dataset (HYRAS). Meteorol. Z. 22(3), 235-256 (2013)

Statistisches Bundesamt: $61 \%$ des Wassers im Jahr 2016 aus Grundwasser gewonnen. Pressemitteilung Nr. 451 vom 21. November 2018 (2018). https://www.destatis.de/DE/Presse/Pressemitteilung en/2018/11/PD18_451_322.html. Zugegriffen: 15.05.2020

Thober, S., Marx, A., Boeing, F.: Auswirkungen der globalen Erwärmung auf hydrologische und agrarische Dürren und Hochwasser 
in Deutschland - Ergebnisse aus dem Projekt HOKLIM: Hochaufgelöste Klimaindikatoren bei einer Erderwärmung von 1.5 Grad. Leipzig (2018). https://www.ufz.de/export/data/2/207531_ HOKLIM_Broschüre_final.pdf. Zugegriffen: 15.05.2020

Umweltministerium Baden-Württemberg, Landesanstalt für Umwelt, Messungen und Naturschutz Baden-Württemberg (Hrsg.): WaBoA: Wasser- und Bodenatlas Baden-Württemberg, 4. Aufl. Umweltministerium Baden-Württemberg und Landesanstalt für Umwelt, Messungen und Naturschutz Baden-Württemberg, Stuttgart, Karlsruhe (2012)
Watzel, R.: Sicherung der Lebensgrundlage Grundwasser - eine globale Gemeinschaftsaufgabe. Grundwasser - Zeitschrift der Fachsektion Hydrogeologie 25(3), 187-188 (2020)

Hinweis des Verlags Der Verlag bleibt in Hinblick auf geografische Zuordnungen und Gebietsbezeichnungen in veröffentlichten Karten und Institutsadressen neutral. 